\title{
Spiral wire localization of lung nodules: procedure effectiveness and oncological usefulness
}

\author{
Miriam Patella ${ }^{1}$, Dario Alberto Bartolucci ${ }^{2}$, Francesco Mongelli ${ }^{1}$, Roberto Cartolari ${ }^{2}$, \\ Eleonora Maddalena Minerva ${ }^{1}$, Rolf Inderbitzi ${ }^{1}$, Stefano Cafarotti ${ }^{1}$ \\ ${ }^{1}$ Department of Thoracic Surgery, ${ }^{2}$ Service of Radiology, San Giovanni Hospital, Bellinzona, Switzerland \\ Contributions: (I) Conception and design: M Patella, DA Bartolucci, R Cartolari, S Cafarotti; (II) Administrative support: R Inderbitzi; (III) Provision \\ of study materials and patients: M Patella, DA Bartolucci, R Cartolari, R Inderbitzi, S Cafarotti; (IV) Collection and assembly of data: M Patella, DA \\ Bartolucci, F Mongelli, EM Minerva; (V) Data analysis and interpretation: M Patella, F Mongelli, R Inderbitzi; (VI) Manuscript writing: All authors; \\ (VII) Final approval of manuscript: All authors. \\ Correspondence to: Miriam Patella, MD. Department of Thoracic Surgery, San Giovanni Hospital, Via Ospedale, 6500, Bellinzona, Switzerland. \\ Email: miriam.patella@eoc.ch.
}

Background: In the last years, a large number of techniques and devices for localizing small pulmonary nodules prior to resection have been developed with the aim of facilitating minimally invasive surgery (VATS). However, each device presents pros and cons and there is no unanimous consensus. We report our experience with an uncommon wire system with spiral shape for percutaneous marking.

Methods: We recorded 102 consecutive CT-guided spiral wire localizations in our Institution, and we evaluated the efficacy of the method according to 4 success rates (SR): (I) successful targeting rate (SR-1): number of successful targeting procedures/number of all localizations; (II) successful localization in operative field (SR-2): (number of successful targeting procedures — number of dislodgements in operative field)/ number of all localizations; (III) successful VATS rate (SR-3): number of successful VATS procedures/(number of localizations-number of thoracotomies not due to wire dislocation); (IV) successful curative rate (SR-4): number of neoplastic nodules resected with curative intent with free margins (R0) on definitive tissue diagnosis/ number of neoplastic nodules resected with curative intent. Complications rate was recorded as well.

Results: SR-1: 100\%, SR-2: 97.1\%, SR-3: 100\%, SR-4: 100\%. Asymptomatic pneumothorax and minimal parenchymal hemorrhage were observed in 5 (4.9\%) and 19 (18.6\%) cases, respectively.

Conclusions: Spiral wire localization showed very good results in terms of feasibility, stability in operative field and contributed to effective use of VATS during wedge resection performed for malignant nodules. In the era of widespread radiological investigations (as it is happening in lung cancer screening) and evolutions in cancer treatments, this appears to be clinically relevant.

Keywords: Small pulmonary nodules; localization; lung resection; video-assisted thoracic surgery (VATS)

Submitted Jul 14, 2019. Accepted for publication Sep 27, 2019.

doi: $10.21037 /$ jtd.2019.11.74

View this article at: http://dx.doi.org/10.21037/jtd.2019.11.74

\section{Introduction}

Developments in thoracic surgery, oncology and epidemiology have broadened the indications for lung nodules resection. Surgeons are required to obtain definitive tissue diagnosis or to treat oncological diseases. Video-assisted thoracic surgery (VATS) minimizes surgical trauma and morbidity and represents an optimal choice. Nevertheless, instrumental palpation of the parenchyma is often insufficient to localize target lesions (1).

A large number of localization techniques and devices have been recently developed to facilitate these procedures. In fact, pre-operative nodule marking allows precise localization and speeds-up surgical intervention while 


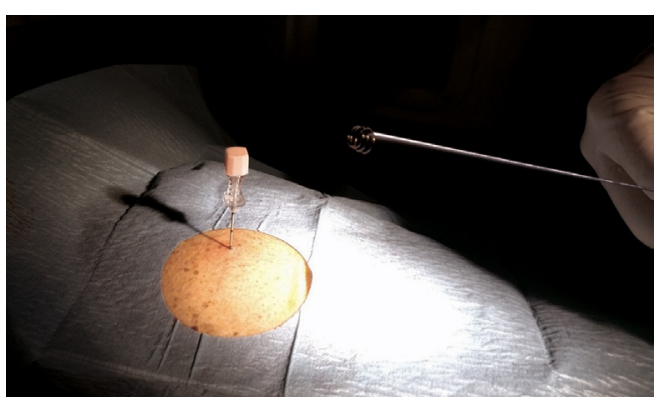

Figure 1 Spiral wire localization procedure: the nitinol wire is introduced through an 18-gauge needle under CT-fluoroscopy.

inadequate localization during surgical procedures can lead to prolonged operative time, conversion to thoracotomy or even failure in resecting target nodules (1-4).

Definitively, the best method has to be precisely feasible, stable enough in the context of minimally invasive surgery, and effective in obtaining the desired surgical results. To date, no flawless technique has been identified and each existing device presents pros and cons. The most widely used is the CT-guided wire localization (5). It is considered both safe and effective, but its major limitation is the risk of dislodgement prior to or during the operative phase, with varying success rates reported (5).

The vast majority of studies on wire localization consider the use of hook-wires which are shaped with single or double thorn $(1,2,6-20)$, with low anchorage capacity of the hook (21). In our institution, we currently use a spiral wire with three-helical shape, strong anchoring power and atraumatic tip (21). The stability during VATS procedures seems to be increased thanks to its shape; this decreases the rate of dislodgement, and contributes to more linear technical movements in the operating room.

We performed a retrospective analysis of our experience aiming to verify these hypotheses. We assessed the efficacy of wire localization throughout different aspects for a comprehensive evaluation of the technique. Particular attention has been paid to evaluating this method in the context of oncological resections.

\section{Methods}

This study was conducted in accordance with the amended Declaration of Helsinki. Ethics Committee of Canton Ticino approved the protocol (number of protocol 201801217/CE3381), and written informed consent was obtained from all patients.

\section{Patients' characteristics}

Between October 2015 and July 2018, spiral wire localization was performed for 102 pulmonary nodules in 93 consecutive patients at San Giovanni Hospital, Bellinzona, Switzerland. Nine patients had multiple localizations, of which two were bilateral. Indication for pre-operative localization was assessed by a senior thoracic surgeon based on nodule characteristics (solid vs. groundglass) and dimensions, position within lung parenchyma and surgical resection intent (diagnostic with or without frozen section vs. curative). Overall, for ground-glass opacities, lesions smaller than $5 \mathrm{~mm}$ and nodules located $>5 \mathrm{~mm}$ below the pleural surface, the indication for localization was absolute. Target nodules planned for curative resection were preferably localized compared to lesions suitable for intraoperative frozen section. Risks and benefits were explained in the outpatient clinic and written informed consent was obtained.

\section{Marker placement and surgical procedure}

Planning of marker vector and positioning were discussed amongst surgeons and interventional radiologists. The positioning of the spiral wire markers through the parenchyma was planned in such a way as to intraoperatively provide maximal ergonomic efficiency. Both the pathway and the tip depth were designed to help complete resection of nodules (i.e., the tip positioned deeper than the nodule to ensure inclusion in the planned suture line).

The procedures were performed the same day of the surgery in the Radiology Department, under CTfluoroscopy, using advanced reprocessing tools to assist the needle insertion. The marker system (Lung Marker System; Somatex Medical Technologies, Berlin, Germany) consisted of an 18-gauge introducer needle (length $15 \mathrm{~cm}$ ) and a nitinol wire provided with a spiral-shaped memory (Figure 1). An angio-suite with Cone Beam CT (CBCT) tool (Allura XPer FD20 C, Philips, Koninklijke, The Netherlands) was used.

Anterior, lateral, or posterior access was chosen, depending on the nodule position, and was planned in consultation with the thoracic surgeon. A CBCT was acquired to identify the nodule. Using an advanced workstation (Interventional WorkStation/IWS, Philips, Koninklijke, The Netherlands), volume data were processed and the needle pathway was planned with dedicated software.

The introducer was inserted under fluoroscopic control 

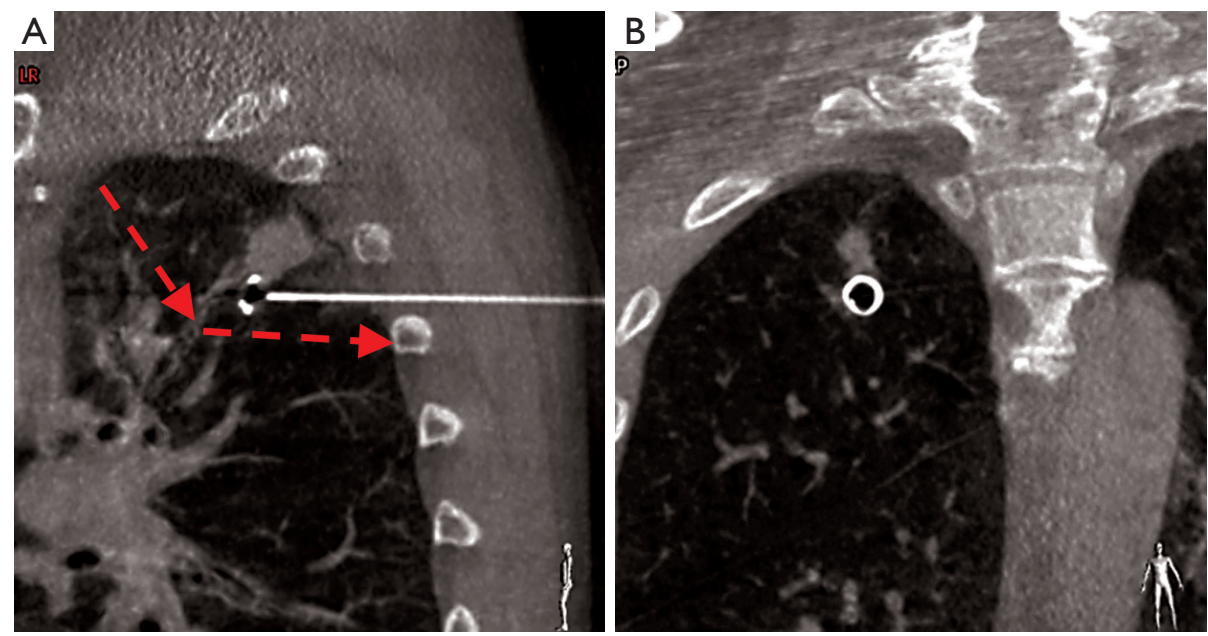

Figure 2 After the procedure, a CT scan is obtained to control correct positioning and check for complications. Right upper lobe nodule with elongated shape. Tip of the wire positioned at the deepest margin of the lesion. Red arrows represent the planned resection line.
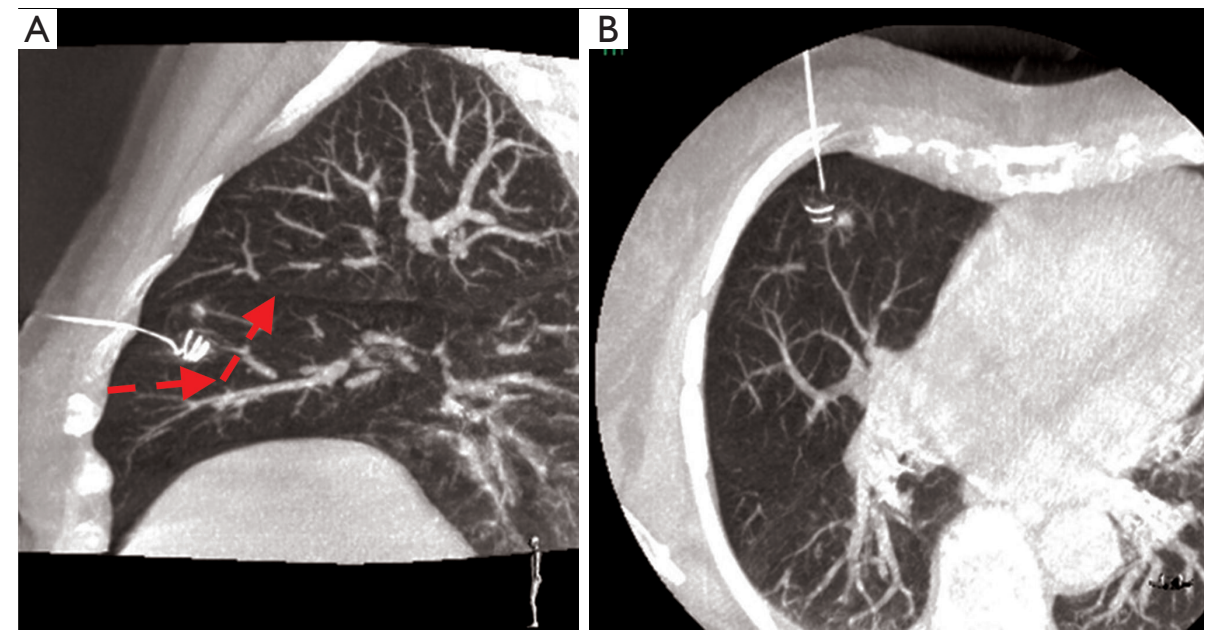

Figure 3 Middle lobe nodule. Wedge resection planned having the horizontal fissure as cranial margin. The lower margin and the depth of the resection are driven by the marker. Red arrows represent the planned resection line.

with a 3D superimposed in an angio-suite until reaching the desired position. The wire was then deployed and a final CT scan was obtained to check for complications. The external part was loosely rolled and draped. Figures 2-4 show different examples of successful nodule targeting according to the vector planning of the stapler lines.

Surgery was performed under general anesthesia and one lung-ventilation. VATS technique consisted of two accesses for $7 \mathrm{~mm}$ trocars and one $11.5 \mathrm{~mm}$ access for staplers. Finger palpation of the lung was not possible through the incisions. The wire was identified and pulled gently to stretch the lung, and wedge resections were performed with $45 \mathrm{~mm}$ staplers (Echelon Flex ${ }^{\mathrm{TM}}$, Ethicon, Somerville, NJ, USA). Surgical specimens were extracted with endobags. Patients received standard care including single chest drain connected with digital drain system, respiratory physiotherapy and pain management.

\section{Surgical strategy by resections' intent}

For patients with history of malignancy and pulmonary nodules highly suspicious for metastasis, patients needing 

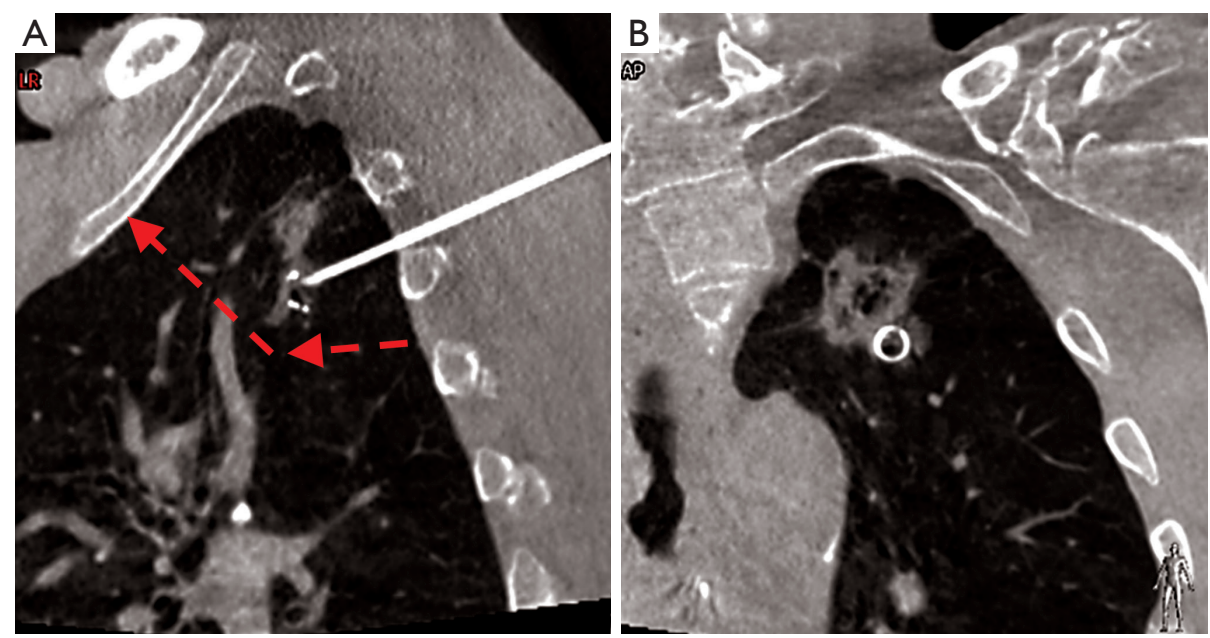

Figure 4 Left upper lobe adenocarcinoma in patient with previous lung cancer and limited pulmonary function. The markers were placed in such a way to ensure free resection margins, post-procedural images were discussed with surgeon. Red arrows represent the planned resection line.

definitive tissue diagnosis, and patients not suitable for any further lung resection, the surgical specimens were sent for final histopathological examination.

For patients with suspected primary lung cancer, eligible for further lung resection, nodules were sent for frozen section and, in cases of positive responses, the indicated anatomical lung resections were performed.

All indications were discussed during multidisciplinary tumor board meetings.

\section{Evaluation of the outcome}

Primary objective of the study was to evaluate the effectiveness of the spiral wire localization procedure. To compare the results of our analysis with the recent literature (5), we defined success rate for subgroups as follows:

(I) success rate (SR)-1: successful targeting rate during localization procedure, defined as the number of successful targeting procedures (wire inserted in the desired position)/number of all localization procedures (5). This rating method refers to feasibility.

(II) SR-2: successful localization rate in the operative field, defined as (the number of successful targeting procedures-the number of dislodgements or misses in the operative field)/the number of all localization procedures (5). It describes the stability of the localization during patients' transport and manipulation of the lung.
(III) SR-3: successful VATS rate, defined as the number of successful VATS procedures/(the number of localization procedures-the number of thoracotomies not due to wire dislocation). This success rate allows the definition of the localization contribution during VATS procedures and, accordingly, the rate of conversion to open surgery due to localization failure.

(IV) SR-4: successful resection rate. This parameter defines the rate of successful resection performed with curative intent, defined as the number of neoplastic nodules resected with curative intent with free margins (R0) on definitive tissue diagnosis/number of neoplastic nodule resected with curative intent.

To investigate this aspect all pathological specimens were reviewed and microscopic (R1) or macroscopic (R2) involvement of the margins by the tumor were recorded as positive $(\mathrm{R}+)$.

As a secondary outcome, we evaluated the complication rate related to the localization.

\section{Statistical analysis}

Outcomes were tested for possible association with patients' characteristics or technical aspects. The following factors were considered as independent variables: age, gender, cancer history, presence of emphysema, nodule size and aspect (solid $v s$. pure or prevalent ground-glass opacities), 
Table 1 Characteristics of patients, nodules, localization and surgical procedures

\begin{tabular}{|c|c|}
\hline Variable & n (\%) \\
\hline \multicolumn{2}{|l|}{ Patient characteristics, $(n=93)$} \\
\hline Age, years & $65.9(8.6)$ \\
\hline Gender male & $56(60.2)$ \\
\hline Cancer history & $57(61.3)$ \\
\hline Emphysema & $51(54.8)$ \\
\hline \multicolumn{2}{|l|}{ Nodule characteristics, $(n=102)$} \\
\hline Size, mm & $11.4(5.4)$ \\
\hline Solid vs. GGO & $80(78.4)$ \\
\hline Upper + middle vs. lower lobe & $52(51.0)$ \\
\hline Distance to the pleura, $\mathrm{mm}$ & $23.1(5.4)$ \\
\hline \multicolumn{2}{|l|}{ Localization procedures, $(n=102)$} \\
\hline Distance skin-wire tip, mm & $79.3(23.0)$ \\
\hline Distance pleura-wire tip, $\mathrm{mm}$ & $34.4(14.5)$ \\
\hline Distance skin-pleura, mm & $44.8(17.5)$ \\
\hline Multiple & $9(8.8)$ \\
\hline Bilateral & $2(2.0)$ \\
\hline Complications & $24(23.5)$ \\
\hline Pneumothorax & $5(4.9)$ \\
\hline Hemorrhage & $19(18.6)$ \\
\hline \multicolumn{2}{|l|}{ Surgical procedures } \\
\hline Dislocation & $3(2.9)$ \\
\hline VATS & $96(94.1)$ \\
\hline Conversion due to dislocation & 0 \\
\hline Frozen section & $17(16.7)$ \\
\hline
\end{tabular}

Results are expressed as mean \pm standard deviation, unless otherwise specified. GGO, ground-glass opacity; VATS, videoassisted thoracoscopic surgery.

location of the nodule (upper lobes + middle lobe $v s$. lower lobes), distance of the nodule from the nearest pleura, distance skin-wire tip, distance pleura-wire tip, distance skin-wire tip, multiple localization procedures, bilateral localizations. Continuous variables were analyzed using the Student $t$-test; categorical variables were tested by means of Fisher's exact test (in case the number of observations was less than 10 in at least one cell) or Chi-squared test. For outcomes showing less than 10 failure events, simple logistic regression analysis was performed. For outcomes showing at least 10 failure events, variables that were statistically significant at univariable analysis were subsequently entered as independent variables in a stepwise logistic regression analysis with backward elimination with entry criterion set at $\mathrm{P}=0.1$ and retention criterion $\mathrm{P}=0.1$.

The level of significance was set at 0.05 , all statistical analysis were performed using STATA software (StataCorp. 2017. Stata Statistical Software: Release 15. College Station, TX, USA: StataCorp LLC) without correction for multiple testing.

Characteristics of patients, nodules, localization and surgical procedures are listed in Table 1.

\section{Results}

In all 102 cases, the localization was successful in the first attempt (SR-1: 100\%). Partial dislocation of the spiral wire was observed in 3 cases during surgical procedure (SR-2: 97.1\%). The wire was never completely dislodged out of the lung parenchyma, but we observed partial retraction of the tip from the original position. Statistical analysis showed no factors associated to SR-2, except for age, being 65.6 \pm 8.5 vs. $77.7 \pm 4.2$ years in the "no dislocation" and "dislocation" groups, respectively [odds ratio (OR): 1.63; $95 \%$ confidence interval (CI), 1.05-2.52, $\mathrm{P}=0.03]$. Conversion to open thoracotomy was not necessary for any of these patients, but VATS wedge resection was successfully performed (SR-3: 100\%). Considering the partial dislocation of the wire, the surgeon extended the resection deeper into lung parenchyma, following the direction offered by the wire. To ensure complete resection of the target nodule in these cases, a frozen section was requested intraoperatively. Six thoracotomies were performed due to extensive adhesions, the impossibility of maintaining single lung ventilation, or proximity of the nodule to the pulmonary artery.

Fresh frozen section was performed in 17 cases and 15 anatomical lung resections (9 lobectomies and 6 segmentectomies) were performed during the same operation, because of primary lung cancer diagnosis. Additional 15 anatomical lung resections (15 lobectomies) were performed later, according to the definitive tissue diagnosis. In these 15 cases, the frozen section was considered insufficient to differentiate primary lung cancer from metastasis of synchronous or metachronous diseases (i.e., previous HPV positive laryngeal cancer, impossibility to discriminate from synchronous contralateral lung cancer and oligometastatic disease). Some patients expressed the 
Table 2 Final results of surgical procedures

\begin{tabular}{lc}
\hline Variable & $\mathrm{n}(\%)$ \\
\hline Target nodule resected & $102(100.0)$ \\
Malignant histology & $78(76.5)$ \\
Wedge with curative intent & $48(61.5)$ \\
Resection margins R0 & $48(100.0)$ \\
Metastasis & $33(68.8)$ \\
Primary lung cancer & $15(31.3)$ \\
Anatomical lung resection after wedge & $30(38.5)$ \\
Lobectomy & $24(80.0)$ \\
Segmentectomy & $6(20.0)$ \\
Benign histology & $24(23.5)$ \\
\hline
\end{tabular}

wish to undergo only the diagnostic wedge resection and postpone the eventual anatomical lung resection. Two patients had a short time-frame for concomitant oncologic treatments (acute leukemia, pancreatic tumor). Moreover, in some cases, the pathologists were not comfortable in expressing a definitive tissue diagnosis on a frozen section.

Overall, wedge resection was performed with curative intent in 48 cases ( $47 \%)$. Thirty-three patients had metastasis from distant organs or recurrence of lung cancer, while in 15 patients with a diagnosis of primary lung cancer, there was no indication for completion lung resection due to limited pulmonary reserve and/or T1 specific adenocarcinoma histologies [adenocarcinoma in situ, minimally invasive adenocarcinoma and lepidic predominant adenocarcinoma, defined according to the IASLC/ATS/ERS Classification (22)]. All the specimens were $\mathrm{R} 0$ at definitive tissue diagnosis (SR-4: 100\%). All of them showed a resection margin $>10 \mathrm{~mm}$. Twenty-four nodules were benign (23.5\%).

Details of final surgical results are listed in Table 2.

Complications after wire placement occurred after $24(23.5 \%)$ procedures. We observed 5 (4.9\%) small asymptomatic pneumothoraces not requiring intervention and $19(18.6 \%)$ mild pulmonary hemorrhages with no hemoptysis. No haemothorax or air embolism were observed.

Univariate analysis revealed a positive association between pneumothorax and multiple localizations in the same lung (OR 8.2; 95\% CI, 1.26-53.3, $\mathrm{P}=0.03$ ). The univariate analysis showed a statistically significant association between hemorrhage and male gender
$(\mathrm{P}=0.02)$, the diameter of the nodule $(\mathrm{P}=0.02)$, distance of the nodule from the nearest pleura $(\mathrm{P}=0.02)$, length of the intrapulmonary pathway of wires $(\mathrm{P}=0.004)$ and total distance skin-wire tip $(\mathrm{P}=0.01)$. Multivariate analysis confirmed only male gender (OR 4.07; 95\% CI, 1.06-15.60, $\mathrm{P}=0.04)$ and length of intrapulmonary wire pathway (OR 1.05; 95\% CI, 1.01-1.08, $\mathrm{P}=0.01$ ) to be statistically significant independent predictors of hemorrhage.

\section{Discussion}

Our study on CT-guided spiral wire localization of pulmonary nodules prior to wedge resection showed very good results in terms of feasibility, stability in operative field, and contribution to effective use of VATS during wedge resection performed for malignant nodules.

In the last decades, different concurrent factors have widened the indications for surgical resection of lung nodules. The widespread use of radiological imaging, as is happening with the implementation of lung cancer screening programs, has multiplied the findings of undetermined pulmonary nodules in asymptomatic patients (23). Developments in treatments for advanced lung cancer suggest the need for histological samples to guide the therapy (24). Ongoing evidence in early stage lung cancer favors lungspearing approaches (25) and pulmonary metastasis from distant organs may benefit from excision (26). Thus, thoracic surgery patients might present with poor general conditions, as occurs in metastatic cancers, or in patients in extremely good health as those referred during screening programs often are. These two extremes need careful preservation and require a minimally invasive surgical approach with very high diagnostic and therapeutic yield. VATS represents the best technique in this context, given its low morbidity rate (27). However, instrumental palpation may not be sufficient to identify small nodules or ground-glass opacities (1). Many tools have been developed to overcome this limitation: image-guided surgery, injection of liquid material, and placement of percutaneous wires (28). The use of wires, with its several variants, is the oldest and most common technique. Spiral wires have been previously described with promising results (29-31). Nevertheless, the English literature on this device is inconsistent with very few reports. In fact, the vast majority of the studies conducted on the wire localization report the use of hook wires with one or two thorns (1,2,6-20). A recent review on the different localization systems (5) analyzed 46 studies, among which 30 used percutaneous wire, 9 microcoils, 
and 7 lipiodol, concluding that wire localization yields the lowest success rate due to dislodgement, and has the highest rate of complications. Out of the 30 studies on wire localization, only two used spiral wires, thus, results may not be strictly reliable in terms of this specific technique. In fact, the rate of dislodgement has been reported to be up to $47 \%$ (32).

Our experience suggests a high feasibility of the spiral wire technique with an SR-1 of $100 \%$ and high stability in the operative field with an SR-2 of $97.1 \%$. These results are better than those reported in the literature on hook wires (5), suggesting their major limitations were overcome.

SR-3 describes the high efficacy of spiral wires during minimally invasive surgery. With this specific definition, we wanted to focus on the conversion rate due to dislodgement of the wire and consequently failure of the procedure. In fact, conversion from VATS to open thoracotomy is not always due to failure in localizing the target nodule during the exploration of the chest cavity. In our series, six thoracotomies were performed for issues not related to the localization of the nodules. We believe that focusing on the thoracotomies performed due to the localization failure, rather than on the conversion rate, is more informative of the device performance.

As reported by other authors (29-31), we used the portion of the wire located outside the thoracic wall to apply a gentle traction: this allowed the exact placement of the stapler with minimal loss of healthy lung parenchyma without compromising the oncological outcome. Moreover, the extreme elasticity of the nitinol gives to spiral wires the capacity to be deformed by the staplers without being cut. When the straight part of the wire is accidentally included inside the stapler, the lung is cut while the wire is deformed and re-straightened after opening the stapler. In clinical practice, the surgeon can clearly see the wire coming out of the stapled lung if the resection does not include completely the marker avoiding the need for fluoroscopy use during the surgery.

SR-4 acquires specific relevance when surgical resection is performed with curative intent. For metastasectomies, resection of pulmonary nodules in patients with profoundly limited pulmonary reserve (33) or resection of some $\mathrm{T} 1$ primary lung cancer (25), obtaining free margins is a mandatory part of the treatment. This aspect of the effectiveness of wire localization has not been extensively investigated. Even though frozen section has been used in many studies with the intent of directing further anatomic lung resection, very few looked specifically at resection margins $(1,3,17)$. Moreover, frozen section is time-consuming and not always feasible (34) as it could be insufficient in discriminating primary lung cancers from metastatic nodules.

In our study, we reviewed all definitive pathological reports of surgical specimens for the evaluation of resection margins in case of malignancy. Looking at metastatic lesions resected, any of them was shown to be $\mathrm{R}+$. In regards to primary lung cancers treated with wedge resection because of histology/stage or because of limited pulmonary reserve, none resulted as R+. Altogether, resections performed with curative intent had an SR-4 of $100 \%$, indicating spiral wire localization as highly effective in these patients and suggesting that routine frozen section may not be needed. Moreover, all the nodules were resected with a distance from the margins of at least $10 \mathrm{~mm}$.

A secondary outcome of this study was the incidence of localization-related complications. Overall, the complications rate was $23.2 \%$, with 5 pneumothoraces and 19 mild pulmonary hemorrhages. None of the patients needed chest tube insertions, nor did the hematoma impair the surgical procedure or cause hemoptysis. The rate of pneumothoraces was lower than that reported for hook wires, microcoils and lipiodol (5), while the rate of hemorrhages was higher than the other methods. As expected, the risk of hemorrhage increased with the length of the intrapulmonary wire pathway and, in our experience, we did not always follow the shortest route to localize target nodules. However, we believe that having the marker placed in a comfortable position for the surgeon may be important to achieving a good resection as shown by success rates 3 and 4 . Moreover, placing the wire's tip beyond the target nodule and including it in the wedge, supports complete resection with R0 margins. Preprocedure and post-procedure discussion amongst the specialists involved, helps plan the strategy in the operative field. In this sense, the shorter route may not necessarily be the best one. The choice of utilizing the angio-suite rather than the regular CT scan, was also made to improve and strengthen this collaboration. The prone position, the supine position and all the intermediate positions between these, were used to improve the chances to obtain a good marker release. Moreover, the use of the advanced tools available in a modern angio-suite allow more free pathways of needle insertion, which are restricted to vertical routes in CT scan localizations. The ability to follow craniocaudal pathways (and vice versa) and steep inclination of the needle insertion was crucial to avoid inconvenient or 
dangerous paths. Typically, this feature was used to avoid fissures or vascular structures, or to reach a proper position of the marker when the shortest route was prevented by skeletal structures.

Some authors reported an improvement in the safety of the wire localization with the use of the hybrid theatre, which allows real-time marking placement and surgery to be performed in the same room. Chao and colleagues (35) mentioned a reduction of the patient time at risk (interval from completion of localization to skin incision). However, they reported a significant increase in general anesthesia time and in the operation theatre (OT) utilization time. Specifically, with the use of intraoperative localization, the latter increased by about 60 minutes on average. Even thought our study focused on the clinical aspects of spiral wire localization, and the safety of patients is primarily important, this OT utilization time is not sustainable. In our experience, a standard localization procedure takes about 15 minutes with a total angio-suite utilization time of 30 minutes. On average, a wedge resection takes 30-45 minutes skin-to-skin with a total OT utilization time of about 60-70 minutes. Considering that 1-hour OT utilization has an overall cost of 1,350 Euro, the intraoperative localization procedure would not be cost-effective.

Our study has some limitations. As single institution experience with one device, we could only compare our results with the published literature, as opposed to comparisons with on-field practice of other techniques. In addition, because our team is composed of very experienced interventional radiologists in thoracic and collaboration occurs with the surgical team on a daily basis, some of the study definitions are not standardized as the planning of the markers is designed on each patient according to a tailored approach. We found that a constructive discussion between the specialists involved produces the best results. This is also true for the interval between the localization and the operation: during this time, patients are sent to the preanesthetic room and prepared for the surgery. In this way, the anesthetic team utilizes the "dead time" improving efficiency and monitoring the patients after the invasive procedure. Unfortunately, we have a lack of data regarding the localization-to-theatre interval, which could add more value to the utilization of resources.

Finally, because failure events were quite rare, the statistical analysis is limited. Multivariate analysis was performed only for the event "hemorrhage" and the ORs were quite low, even if significant. Broader experience with this device may provide more accurate results.

\section{Conclusions}

In conclusion, despite the limitations of the study, spiral wires appear to be very promising in the management of small pulmonary nodules and ground-glass opacities. Not only could they help the localization of lesions, but they could also support the use of VATS and facilitate oncological correct resections. In the era of widespread radiological investigations and evolutions in cancer treatments, this appears to be clinically relevant.

\section{Acknowledgments}

None.

\section{Footnote}

Conflicts of Interest: The authors have no conflicts of interest to declare.

Ethical Statement: The authors are accountable for all aspects of the work in ensuring that questions related to the accuracy or integrity of any part of the work are appropriately investigated and resolved. This study was conducted in accordance with the amended Declaration of Helsinki. Ethics Committee of Canton Ticino approved the protocol (number of protocol 2018-01217/CE3381), and written informed consent was obtained from all patients.

\section{References}

1. Nakashima S, Watanabe A, Obama T, et al. Need for preoperative computed tomography-guided localization in video-assisted thoracoscopic surgery pulmonary resections of metastatic pulmonary nodules. Ann Thorac Surg 2010;89:212-8.

2. Seo JM, Lee HY, Kim HK, et al. Factors determining successful computed tomography-guided localization of lung nodules. J Thorac Cardiovasc Surg 2012;143:809-14.

3. Miyoshi K, Toyooka S, Gobara H, et al. Clinical outcomes of short hook wire and suture marking system in thoracoscopic resection for pulmonary nodules. Eur J Cardiothorac Surg 2009;36:378-82.

4. Hanauer M, Perentes JY, Krueger T, et al. Pre-operative localization of solitary pulmonary nodules with computed tomography-guided hookwire: report of 181 patients. J Cardiothorac Surg 2016;11:5.

5. Park CH, Han K, Hur J, et al. Comparative effectiveness 
and safety of preoperative lung localization for pulmonary nodules: a systematic review and meta-analysis. Chest 2017;151:316-28.

6. Wicky S, Dusmet M, Doenz F, et al. Computed tomography-guided localization of small lung nodules before video-assisted resection: experience with an efficient hook-wire system. J Thorac Cardiovasc Surg 2002;124:401-3.

7. Paci M, Annessi V, Giovanardi F, et al. Preoperative localization of indeterminate pulmonary nodules before videothoracoscopic resection. Surg Endosc 2002;16:509-11.

8. Kanazawa S, Ando A, Yasui K, et al. Localization of pulmonary nodules for thoracoscopic resection: experience with a system using a short hookwire and suture. AJR Am J Roentgenol 1998;170:332-4.

9. Chen S, Zhou J, Zhang J, et al. Video-assisted thoracoscopic solitary pulmonary nodule resection after CT-guided hookwire localization: 43 cases report and literature review. Surg Endosc 2011;25:1723-9.

10. Suzuki K, Shimohira M, Hashizume T, et al. Usefulness of CT-guided hookwire marking before video-assisted thoracoscopic surgery for small pulmonary lesions. J Med Imaging Radiat Oncol 2014;58:657-62.

11. Iguchi T, Hiraki T, Gobara H, et al. CT fluoroscopyguided preoperative short hook wire placement for small pulmonary lesions: evaluation of safety and identification of risk factors for pneumothorax. Eur Radiol 2016;26:114-21.

12. Ciriaco P, Negri G, Puglisi A, et al. Video-assisted thoracoscopic surgery for pulmonary nodules: rationale for preoperative computed tomography-guided hookwire localization. Eur J Cardiothorac Surg 2004;25:429-33.

13. Ichinose J, Kohno T, Fujimori S, et al. Efficacy and complications of computed tomography-guided hook wire localization. Ann Thorac Surg 2013;96:1203-8.

14. Xu X, Yao Y, Shen Y, et al. Clinical Analysis of Percutaneous Computed Tomography-Guided Hook Wire Localization of 168 Small Pulmonary Nodules. Ann Thorac Surg 2015;100:1861-7.

15. Li W, Wang Y, He X, et al. Combination of CT-guided hookwire localization and video-assisted thoracoscopic surgery for pulmonary nodular lesions: Analysis of 103 patients. Oncol Lett 2012;4:824-8.

16. Iguchi T, Hiraki T, Gobara H at al. Simultaneous Multiple Preoperative Localizations of Small Pulmonary Lesions Using a Short Hook Wire and Suture System. Cardiovasc Intervent Radiol 2015;38:971-6.
17. Gonfiotti A, Davini F, Vaggelli L, et al. Thoracoscopic localization techniques for patients with solitary pulmonary nodule: hookwire versus radio-guided surgery. Eur J Cardiothorac Surg 2007;32:843-7.

18. Iguchi T, Hiraki T, Gobara H, et al. Transfissural route used for preoperative localization of small pulmonary lesions with a short hook wire and suture system. Cardiovasc Intervent Radiol 2015;38:222-6.

19. Pittet O, Christodoulou M, Pezzetta E, et al. Videoassisted thoracoscopic resection of a small pulmonary nodule after computed tomography-guided localization with a hook-wire system. Experience in 45 consecutive patients. World J Surg 2007;31:575-8.

20. Chen YR, Yeow KM, Lee JY, et al. CT-guided hook wire localization of subpleural lung lesions for video-assisted thoracoscopic surgery (VATS). J Formos Med Assoc 2007;106:911-8.

21. Partik BL, Leung AN, Müller MR, et al. Using a dedicated lung-marker system for localization of pulmonary nodules before thoracoscopic surgery. AJR Am J Roentgenol 2003;180:805-9.

22. Travis WD, Brambilla E, Noguchi $M$, et al. International association for the study of lung cancer/american thoracic society/european respiratory society international multidisciplinary classification of lung adenocarcinoma. J Thorac Oncol 2011;6:244-85.

23. Qian F, Yang W, Chen Q, et al. Screening for early stage lung cancer and its correlation with lung nodule detection. J Thorac Dis 2018;10:S846-59.

24. Ko R, Kenmotsu H, Serizawa M, et al. Frequency of EGFR T790M mutation and multimutational profiles of rebiopsy samples from non-small cell lung cancer developing acquired resistance to EGFR tyrosine kinase inhibitors in Japanese patients. BMC Cancer 2016;16:864.

25. Ito H, Nakayama $H$, Murakami S, et al. Does the histologic predominance of pathological stage IA lung adenocarcinoma influence the extent of resection? Gen Thorac Cardiovasc Surg 2017;65:512-8.

26. Higashiyama M, Tokunaga T, Nakagiri T, et al. Pulmonary metastasectomy: outcomes and issues according to the type of surgical resection. Gen Thorac Cardiovasc Surg 2015;63:320-30.

27. Begum SS, Papagiannopoulos K, Falcoz PE, et al. Outcome after video-assisted thoracoscopic surgery and open pulmonary lobectomy in patients with low VO2 max: a case-matched analysis from the ESTS database. Eur J Cardiothorac Surg 2016;49:1054-8; discussion 1058.

28. Dendo S, Kanazawa S, Ando A, et al. Preoperative 
localization of small pulmonary lesions with a short hook wire and suture system: experience with 168 procedures. Radiology 2002;225:511-8.

29. Torre M, Ferraroli GM, Vanzulli A, et al. A new safe and stable spiral wire needle for thoracoscopic resection of lung nodules. Chest 2004;125:2289-93.

30. Hänninen EL, Langrehr J, Raakow R, et al. Computed tomography-guided pulmonary nodule localization before thoracoscopic resection. Acta Radiol 2004;45:284-8.

31. Eichfeld U, Dietrich A, Ott R, et al. Video-assisted thoracoscopic surgery for pulmonary nodules after computed tomography-guided marking with a spiral wire. Ann Thorac Surg 2005;79:313-6; discussion 316-7.

32. Bernard A. Resection of pulmonary nodules using video-

Cite this article as: Patella M, Bartolucci DA, Mongelli F, Cartolari R, Minerva EM, Inderbitzi R, Cafarotti S. Spiral wire localization of lung nodules: procedure effectiveness and oncological usefulness. J Thorac Dis 2019;11(12):5237-5246. doi: $10.21037 /$ jtd.2019.11.74 assisted thoracic surgery. The Thorax Group. Ann Thorac Surg 1996;61:202-4; discussion 204-5.

33. Harrison S, Stiles B, Altorki N. What is the role of wedge resection for T1a lung cancer? J Thorac Dis 2018;10:S1157-62.

34. Gupta R, McKenna R Jr, Marchevsky AM. Lessons learned from mistakes and deferrals in the frozen section diagnosis of bronchioloalveolar carcinoma and well-differentiated pulmonary adenocarcinoma: an evidence-based pathology approach. Am J Clin Pathol 2008;130:11-20.

35. Chao YK, Pan K, Wen CT, et al. A comparison of efficacy and safety of preoperative versus intraoperative computed tomography-guided thoracoscopic lung resection. J Thorac Cardiovasc Surg 2018;156:1974-83. 